\title{
Dermoscopy of dermatofibromas: a new perspective
}

\author{
Sema Aytekin ${ }^{1}$, Emre Kaynak ${ }^{2}$, and Erhan Ayhan ${ }^{3}$ \\ ${ }^{1}$ Istsanbul Haydarpasa Numune Training and Research Hospital \\ ${ }^{2}$ Doku Medical Center \\ ${ }^{3}$ Affiliation not available
}

March 14, 2021

\begin{abstract}
Introduction: In dermoscopic studies on dermatofibromas, some publications divide the appearance of lesions into standardized patterns, and some publications classify the clinical appearance of the lesions by comparing them with existing dermatological lesions. Objectives: This study aims to re-evaluate the dermoscopic findings and patterns of dermatofibromas from a different perspective. Methods: In this study, 142 lesions of 72 patients were evaluated dermoscopically and their patterns were schematized. Results: In our study, a total of 15 patterns consisting of main and sub-patterns were created. The most common patterns we detected were pattern 1 (1a: $13.4 \%, 1 \mathrm{~b}: 8.5 \%)$, pattern 8 (8a: $10.6 \%, 8 \mathrm{~b}: 4.2 \%)$, pattern 2 (2a: $9.2 \%, 2 \mathrm{~b}: 4.2 \%)$, respectively. Conclusions: Patterns of DFs were reclassified while preserving basic patterns. We think that the new sub-patterns and schematization with this study can contribute to better understanding of DFs.
\end{abstract}

\section{Dermoscopy of dermatofibromas: a new perspective} ABSTRACT

Aim: In dermoscopic studies on dermatofibromas, some publications divide the appearance of lesions into standardized patterns, and some publications classify the clinical appearance of the lesions by comparing them with existing dermatological lesions. This study aims to re-evaluate the dermoscopic findings and patterns of dermatofibromas from a different perspective.

Materials and Methods: In this study, 142 lesions of 72 patients were evaluated dermoscopically and their patterns were schematized.

Results: In our study, a total of 15 patterns consisting of main and sub-patterns were created. The most common patterns we detected were pattern 1 (1a: $13.4 \%, 1 \mathrm{~b}: 8.5 \%$ ), pattern 8 (8a: $10.6 \%, 8 \mathrm{~b}: 4.2 \%$ ), pattern 2 (2a: $9.2 \%, 2 \mathrm{~b}: 4.2 \%)$, respectively.

Conclusion: Patterns of DFs were reclassified while preserving basic patterns. We think that the new sub-patterns and schematization with this study can contribute to better understanding of DFs.

Keywords: Dermatofibroma, dermoscopy, benign skin neoplasm.

\section{What's knownn}

- Dermatofibromas are common benign skin lesions that can be easily diagnosed clinically in most cases.

- However, it can be clinically difficult to distinguish DFs from other lesions such as dysplastic nevi or malignant melanoma.

- There are 4 main dermoscopic structures in dermatofibromas, mainly peripheral delicate pigment network, central white scar-like patch, white network and homogeneous pigmentation 


\section{What's new}

The patterns of DF's were reclassified by preserving the basic patterns.

The new sub-patterns and schematization with this study can contribute to better understanding of DFs.

\section{INTRODUCTION}

Dermatofibromas (DF) are common benign skin lesions that can be easily diagnosed clinically in most cases. However, it can be clinically difficult to distinguish DFs from other lesions such as dysplastic nevi or malignant melanoma (1).

It is mildly predominant in female patients, but often affects young or middle-aged adults. It reveales as flat, firm, single or multiple papules, plaques or nodules, clinically characterized by a variety of colors from light brown to dark brown, purple, red or yellow (2). It is a soft tissue and bone neoplasm composed of fibroblastic and histiocytic components without nuclear pleomorphism or histological anaplasia $(3,4)$. Dermoscopy (dermatoscopy or epiluminescence microscope) is an in vivo, non-invasive technique that reveals a new dimension of clinical-morphological features in pigmented and non-pigmented skin lesions (2). In previous studies, the presence of pigment network and central white patch has been defined as the typical dermoscopic appearance of DF (3). However, Zaballos et al. identified a total of 11 dermoscopic patterns of DFs, one of which is subtype, including different dermoscopic findings as well as these typical clinical dermoscopic findings (2). The purpose of this prospectively designed study is to re-evaluate the dermoscopic findings and patterns of DFs with a different perspective.

\section{MATERIALS AND METHODS}

The study included 142 lesions of 72 patients, aged 26-79, who were admitted to the dermatology department of Haydarpaşa Numune Training and Research Hospital between 2014-2018 and diagnosed clinically and / or histopathologically (for atypical lesions) as dermatofibroma. Clinical data were recorded for each patient, including age and sex, location of the lesion, and size of the lesion. This study includes three stages including dermatological and dermoscopic examination of lesions, macroscopic and dermoscopic (Dermlite II hybrid M Pocket Epiluminescence Device, Molemax 3 Digital Image Capture) photographing and evaluation of the findings. Macroscopic and dermoscopic pictures of all lesions in the study were taken and data were recorded. Structures classified as vascular and nonvascular were defined dermoscopically. In order to increase the image quality and the visibility of the structures, the contact plate was wetted with saline before dermoscopic pictures were taken. The pressure on the lesion was relieved to prevent collapse of the vascular structures. All datas were uploaded to SPSS 17.0 for Windows statistic application software.

\section{RESULTS}

142 lesions of 72 patients (9 males, 63 females) with a median age of 51 (age range 26-79) were included in the study. The number of patients with a single lesion was 42 . The number of lesions was 2 in 17 patients, 3 in 2 patients, 4 in 7 patients, 5 in 1 patient, 8 in 1 patient, 9 in 1 patient and 10 in 1 patient. Lesion sizes varied between 2-10 $\mathrm{mm}$.

Pigment network was detected in $57 \%$ of DFs. The most common subtype of the pigment network was irregular delicate / asymmetric pigment network with 31 lesions (21.8\%). In addition, peripheral delicate in $17(12 \%)$ lesions, total delicate in $25(17.6 \%)$ lesions, peripheral prominent in 3 (2.1) lesions, total prominent in $1(0.7 \%)$ lesion, irregular prominent in $2(1.4 \%)$ lesions and atypical pigment network was observed in 2 $(1.4 \%)$ lesions.

White scar-like patch was detected in $53(37.3 \%)$ of DFs. The most common subtype of this dermoscopic structure was the central scar-like patch detected in $42(29.6 \%)$ lesions. Multiple irregular type was seen in 11 (7.7) lesions, while the total type was not seen in any lesion.

White network was found in $44(30.9 \%)$ of the lesions. As a subclassification, the central structure was detected in $23(16.2 \%)$ lesions, a total in $3(2.1 \%)$ lesions, crystalline-like (Star-like white patch) in 7 (4.9\%) 
lesions, and white radial streaks in $8(5.6 \%)$ lesions.

Homogeneous areas were seen in $95(66.9 \%)$ of the lesions. Brown homogenous areas were seen in $36(25.4 \%)$ lesions, yellowish homogeneous areas in $18(12.6 \%)$ lesions, multiple colored homogeneous areas (usually redbluish) in 7 (4.9\%) lesions. There were hypopigmented areas in 29 (20.4\%) of the lesions, and perifollicular hypopigmentation in $5(3.5 \%)$.

Vascular structures were observed in $83(58.4 \%)$ of DFs. Erythema, the most common structure, was present in $69(48.5 \%)$ lesions. Dotted vessels were detected in $34(23.9 \%)$ lesions, linear vessels in $13(9.2 \%)$ lesions, hairpin vessels in $3(2.1 \%)$ lesions, glomerular vessels in $2(1.4 \%)$ lesions, comma vessels in $1(0.7 \%)$ lesion, polymorph vessels in $6(4.2 \%)$ lesions (Table 1 ).

All these structures were evaluated and DFs classified as dermoscopically by Zaballos et al. in 2008 were reclassified. Accordingly, a total of 15 patterns consisting of the following sub-patterns were created (Table 2 ).

Pattern 1a: Total delicate network extending the entire lesion.

Pattern 1b: Multiple hypopigmentation / perifollicular hypopigmentation scattered within the total network.

Pattern 2a: Peripheral delicate network surrounding the central white scar-like patch.

Pattern 2b: White network surrounding white scar-like patch in the center and peripheral delicate network outermost surrounding the entire lesion.

Pattern 3a: Peripheral delicate network surrounding the white network in the center.

Pattern 3b: Homogeneous pigmentation surrounding the white network in the center and the peripheral delicate network surrounding the entire lesion outermost.

Pattern 4: Peripheral delicate network surrounding the central homogeneous pigmentation.

Pattern 5: White network extending to the entire lesion.

Pattern 6: Homogeneous pigmentation extending to the entire lesion.

Pattern 7a: White scar-like patch extending to the entire lesion.

Pattern 7b: Multiple scar-like patch spreading over the entire lesion (there may be delicate network around or between the lesion).

Pattern 8a: Homogeneous pigmentation surrounding white scar-like patch in the center.

Pattern 8b: White network surrounding the white scar-like patch in the center and homogeneous pigmentation surrounding the entire lesion outermost.

Pattern 9a: Homogeneous pigmentation surrounding the white network in the center.

Pattern 9b: Homogeneous pigmentation surrounding the crystalline-like white network at the center (Starlike white patch).

Pattern 10: Atypical pigment network, atypical scar-like patch or white network, atypical homogeneous pigmentation, or atypical pattern consisting of irregular distribution of these structures.

The schematic drawing of the patterns of dermatofibromas is shown inFig. 1-2. On the other hand, the pattern equivalents of the dermoscopic images of dermatofibromas are shown in Fig. 3-4 .

\section{DISCUSSION}

There are 4 main dermoscopic structures in dermatofibromas, mainly peripheral delicate pigment network, central white scar-like patch, white network and homogeneous pigmentation. (2). Delicate pigment network 
associated with dermatofibromas is usually thin, light and medium brown, graceful and gradually pale towards the periphery of the lesion (2).

Although the pigment network in DFs is quite similar to that of melanocytes, the pigment network in DF is caused by hyperpigmentation in rete ridges rather than melanocytic proliferation in the basal layer $(2,5)$. Arpaia et al., in their study evaluating $32 \mathrm{DF}$, detected a peripheral delicate pigment network in $31 \%$ of the cases. According to the study, the pigment network was darker in the center, but gradually pale towards the periphery and became brownish thin streaked (6).

In 2006, Ferrari et al. detected a pigment network in $83.3 \%$ of the cases (5). The same author detected the peripheral pigment network in $50.8 \%$ of the cases in 2013. They also found that the peripheral pigment network and central white scar-like patches are associated with female sex and the classic histopathology variant of DFs (7). Kelati et al. found a pigment network in $79 \%$ of the lesions in their study, in which they evaluated $100 \mathrm{DF}$ (3). Agero et al. found that the pigment network rate in DF was $72 \%$ in nonpolarized dermoscopy, $72 \%$ in polarized contact dermoscopy, and $54 \%$ in polarized noncontact dermoscopy (1). Karaarslan et al., on the other hand, observed the pigment network, which they defined as lentigo-like reticular pigmentation, in $23 \%$ of DFs (8). Zaballos et al, in their study evaluating $412 \mathrm{DF}$, detected pigment network in $71.8 \%$ of DFs (2). In our study, we found pigment network in $81(57 \%)$ of DFs. We think that the very different pigment network ratios among previous studies may be due to factors such as the quality of the dermoscopes, contact or non-contact, being polarized or nonpolarized, and the difference between genders in the studies. We also observed that the pigment network was present in the holes in the white network in some lesions.

Another dermoscopic structure frequently observed in dermatofibromas is the central white scar-like patch. These structures are irregular and sharply demarcated white areas. It is characterized histopathologically by distinct fibrosis in the papillary dermis (8). Zaballos et al., in their study evaluating 412 dermatofibroma, detected white scar-like patch mostly localized in the center of the lesion in $57 \%$ of patients (2). In the study of Ferrari et al, this rate was $91.6 \%$ (5). The most common structure that Agero et al. encountered in DFs with non-polarized and polarized contact dermoscopy was the white patch. In this study, they found central white patch in $84 \%$ of dermatofibromas by polarized contact dermoscopy (1). These structures were found to be mostly well and irregularly circumscribed and sometimes in the form of a star. They observed that the central white patch seen in 33 lesions with polarized contact dermoscopy was characterized by shiny white streaks (1). In the study of Arpaia et al, the most common dermoscopic structure was the central white patch with $91.6 \%$ (6). Ferrari et al detected white area in $50 \%$ of DFs (7). In another study, a central white patch was found in $70 \%$ of DFs (3). This structure is thought to be the most stereotypic and widespread manifestation of diffuse fibrous dermatofibroma with peripheral delicate pigment network $(3,5,8)$. In our study, a central white scar-like patch was detected in $37.3 \%$ of DFs. In the periphery of this central structure, the scar structure sometimes turned into a white radial streaks appearance. Some white radial streaks transformed the appearance of the central scar-like patch into a spitzoid pattern (Fig. 5 ).

Zaballos et al. identified a new dermoscopic structure, which they identified mostly in large DFs in 2006 and named as white network (9). This network of white lines and brown holes, seen in $17.7 \%$ of dermatofibromas in the study at 2008, is considered a variation of the classic white scar-like patch with a similar histopathological correlation. It is important to distinguish this structure from the Spitz nevi, dysplastic nevi and negative pigment network in melanomas. In suspected cases or cases with an irregularly distributed white network, excision is required. (2). Ferrari et al. Observed the white network structure in $30 \%$ of DFs in their study in 2013 (7). In our study, white network was detected in $25.3 \%$ of the cases.

The term central white scar-like patch, white network and globule-like structures have been used intertwined in the past. For this reason, we think that the information about globule-like structures from past to present is controversial. Agero et al. did not use the term white network in their study in 2006. Instead, the term globule-like structures was used and this structure was detected $44 \%$ in ratio when polarized contact dermoscopy was used (1). In fact, the globule-like structure appears to be 'holes' in the white network. Ferrari et al. stated in their study in 2000 that there were light and dark brown globules and dots within the central white scar-like patch (5). Karaarslan et al. reported that the main feature of the lesion, which 
they defined as a type 1 lesion, is globules in the scar-like area. They observed this structure at a rate of $38.5 \%$ in their study (8). In our opinion, this term is the name given to the combination of white scar-like patch and a white network. This term has been used before $(5,6,10)$. In fact, it has been stated that these globules are not real globules but 'holes' in the central white fibrotic network (8). Later Zaballos et al. proposed the term central white network for globules within the scar-like area (9). In our study, the "holes" seen in the white network were not evaluated as a globul-like structure and it was observed at a rate of $11.3 \%$ in DFs. Ferrari et al found globul-like structures and ring-like structures, which they consider to be its (globul-like structures) variant, in $27.7 \%$ of the cases. They stated that these structures are not caused by melanocyte nests, but by flat, confluent and hyperpigmented rete ridges. In addition, in this study, unlike other studies, the white network structure and globule-like structures within the central white patch were evaluated separately (7). Flattened and dilated rete ridges are also responsible for small ring-like structures, which are globules with darker peripheral rims (2). Zaballos et al. detected globule-like structures in $41.6 \%$ of the lesions and ring-like structures in $25 \%$ of the lesions. However, according to our observation, globule-like structures close to the center are brown holes in the white network structure. We also think that ring-like structures are a more regular, darker, rounder and thicker lined variant of the peripheral delicate pigment network. Ring-like structures were found $5.6 \%$ in our study.

Another basic dermoscopic finding of dermatofibromas is homogeneous pigmentation. Agero et al observed that brown homogeneous areas, which they classified as the peripheral halo of brown homogeneous pigmentation, were $4 \%$ in nonpolarized dermoscopy and $12 \%$ in polarized noncontact dermoscopy (1). Ferrari et al. observed homogeneous area at a rate of 6.9 in their study in 2013. They found total homogeneous pigmentation most frequently in women and DFs with sebaceous hyperplasia, and peripheral homogeneous pigmentation most frequently in men. (7). Kelati et al. found homogeneous pigmentation at a rate of $36 \%$ in their studies (3). Karaarslan et al. detected homogeneous bluish pigmentation at a rate of $5.9 \%$ and found that this appearance was associated with the hemosiderotic variant of the dermatofibroma (8). On the other hand, Zaballos et al. detected the homogeneous areas at a rate of $24.8 \%$. They observed that $4.4 \%$ of the lesions had yellowish homogeneous areas. (2). In our study, brown homogeneous areas were found at a rate of $25.4 \%$, yellowish homogeneous areas at a rate of $12.6 \%$, multiple colored homogeneous areas at a rate of $4.9 \%$, hypopigmented homogenous areas at a rate of $20.4 \%$ and perifollicular hypopigmentation at a rate of $5.5 \%$.

Vascular structures are one of the criteria used in dermoscopic diagnosis of melanoma and other pigmented and vascular tumor lesions that can mimic melanoma. Ferrari et al. found a reddish coloration around the central white scar-like patch in $29.2 \%$ of the 24 series with dermatofibromas (5). Agero et al. have observed blood vessels in $44 \%$ of dermatofibromas when using polarized contact dermoscopy (1). These authors also found a central pink shade in $10 \%$ of cases and a peripheral diffuse pinkish or reddish area in $28 \%$ (1). Zaballos et al. found vascular structures in $49.5 \%$ of dermatofibromas. The most common vascular structure in this study was erythema (31.5\%) followed by dotted vessels (30.6\%) (2). Ferrari et al. have described 2 dermatofibromas with dotted vessel pattern (11). In 2013, Ferrari et al. detected vascular structure in $50 \%$ of DFs (7). Kelati et al. observed vascular structure in $30 \%$ of the cases. They stated that the most common vascular structures were vessels in the form of dots and commas (3). In our study, the most common vascular structures we found were erythema (48.5\%) and dotted vessels $(23.9 \%)$.

It was evaluated by Zaballos et al. that dermoscopic structures such as scales (12.4\%) and ulceration (4.4\%) detected in dermatofibromas were due to external injuries. It was stated that the yellowish homogeneous areas observed in $4.4 \%$ of the cases corresponded histopathologically to lipophage and Touton giant cells, and these structures were detected in cholestatic and lipidized DF or some early lesions. In addition, fissure and ridges $(2.7 \%)$ and exophytic papillary structures (1.5\%) were observed in DFs with significant epidermal hyperplasia (2). These dermoscopic structures are seen in seborrheic keratoses and less frequently compound and dermal nevi, and represent keratin-filled epidermis invaginations (12-15).

In their dermoscopic study performed by Zaballos et al on 412 DF, they divided DFs into 11 patterns, one of which is subtype, according to their dermoscopic appearance. The most common pattern they observed was 
the combination of the central white scar-like patch and peripheral delicate network, which they classified as pattern $2(34.7 \%)$. The second most frequent was pattern $1(14.6 \%)$ with only the total delicate pigment network (2). Ferrari et al. determined pattern $2(17.7 \%)$ and pattern 6 (total homogeneous pigmentation, $11.5 \%$ ) in their study (when evaluated according to the patterns of Zaballos et al.). (7). In the studies of Kelati and Juliandri, it is seen that the most common pattern is the second pattern $(3,16)$. In our study, the most common patterns we found were pattern $1(21.9 \%)$, pattern $8(14.8 \%)$, pattern $2(13.4 \%)$, respectively. We think that this difference may be due to our reclassification of DFs from a different point of view and racial difference.

As a result, with this study, the patterns of DF's were reclassified by preserving the basic patterns. We think that the new sub-patterns and schematization with this study can contribute to better understanding of DFs.

Conflicts of interest: There are no conflicts of interest.

Financial support: There is no financial support.

Data availability statement: No data are available.

\section{Acknowledgment}

The patients in this manuscript have given written informed consent to publication of their case details.

\section{REFERENCES}

1. Agero AL, Taliercio S, Dusza SW, Salaro C, Chu P, Marghoob AA. Conventional and polarized dermoscopy features of dermatofibroma. Arch Dermatol . 2006;142(11):1431-7.

2. Zaballos P, Puig S, Llambrich A, Malvehy J. Dermoscopy of dermatofibromas: a prospective morphological study of 412 cases. Arch Dermatol . 2008;144(1):75-83

3. Kelati A, Aqil N, Baybay H, Gallouj S, Mernissi FZ. Beyond classic dermoscopic patterns of dermatofibromas: a prospective research study. J Med Case Rep . 2017;11(1):266.

4. Deb P, Singh V, Dutta V, Bhatoe HS, Chandran VM. Primary intracranial benign fibrous histiocytoma: report of an unusual case. JCancer Res Ther . 2014;10(1):200-2.

5. Ferrari A, Soyer P, Peris K, et al. Central white scarlike patch: a dermatoscopic clue for the diagnosis of dermatofibroma. J Am Acad Dermatol 2000;43:1123-5.

6. Arpaia N, Cassano N, Vena GA. Dermoscopic patterns of dermatofibroma.Dermatol Surg . 2005;31(10):1336-9.

7. Ferrari A, Argenziano G, Buccini P, Cota C, Sperduti I, De Simone P, Eibenschutz L, Silipo V, Zalaudek I, Catricalà C. Typical and atypical dermoscopic presentations of dermatofibroma. $J$ Eur Acad Dermatol Venereol . 2013;27(11):1375-80.

8. Kilinc Karaarslan I, Gencoglan G, Akalin T, Ozdemir F. Different dermoscopic faces of dermatofibromas. J Am Acad Dermatol . 2007;57(3):401-6.

9. Zaballos P, Puig S, Malvehy J. Dermoscopy of atypical dermatofibroma: central white network. Arch Dermatol . 2006;142(1):126.

10. Soyer HP, Argenziano G, De Giorgi V, Piccolo D, Delfino M, Ferrari A, et al. Dermoscopy: a tutorial. Milan: EDRA Medical Publishing \& New Media; 2000.

11. Ferrari A, Piccolo D, Fargnoli MC, et al. Cutaneous amelanotic melanoma metastasis and dermatofibromas showing a dotted vascular pattern. Acta Derm Venereol . 2004;84(2):164-5.

12. Malvehy J, Puig S, Argenziano G, et al. Principles of Dermoscopy. Barcelona, Spain: GEDE; 2002.

13. Stolz W, Braun-Falco O, Bilek P, et al. Colour Atlas of Dermatoscopy. Oxford, England: Blackwell Publishing Co; 2002.

14. Soyer H, Argenziano G, Chimenti S, et al. Dermoscopy of Pigmented Skin Lesions: An Atlas Based on the Consensus Net Meeting on Dermoscopy 2000. Milan, Italy: EDRA; 2001.

15. Marghoob AA, Braun RP, Kopf AW. Atlas of Dermoscopy. London, England: Taylor \& Francis Group; 2005 . 
16. Juliandri J, Wang XY, Liu ZJ, Zhang JW, Xu Y. Dermoscopic patterns of dermatofibroma in 72 Chinese patients. Chin Med J (Engl). 2019;132(17):2121-22.

Table Legends

Table 1. Dermoscopic structures of dermatofibromas.

Table 2. Dermoscopic patterns of dermatofibromas

Figure Legends

Figure 1. Schematic view of dermoscopic patterns of dermatofibromas-1 (1a, 1b, 2a, 2b, 3a, 3b, 4, 5 ).

Figure 2. Schematic view of dermoscopic patterns of dermatofibromas-2 ( $6,7 \mathrm{a}, 7 \mathrm{~b}, 8 \mathrm{a}, \mathbf{8 b}, \mathbf{9 a}, \mathbf{9 b}, 10$ ) .

Figure 3. Pattern equivalents of dermoscopic views of dermatofibromas-1 ( $\mathbf{1 a}, \mathbf{1 b}, \mathbf{2 a}, \mathbf{2 b}, \mathbf{3 a}, \mathbf{3 b}, \mathbf{4}, \mathbf{5}$ ) .

Figure 4. . Pattern equivalents of dermoscopic views of dermatofibromas-2 (6, 7b, 8a, 8b, 9a, 9b, 10$)$.

Figure 5. Spitzoid appearance $(\mathbf{a}, \mathbf{b})$ and schematic drawing $(\mathbf{c})$ of the white scar-like patch in the center of the dermatofibroma classified as pattern $8 \mathrm{a}$.

Table 1.

Dermoscopic Structures

Pigment network Peripheral delicate Total delicate Irregular delicate/asymmetric Peripheral prominent Total prominent White scar-like patch Central Total Multiple irregular

White network Central Total Irregular Crystalline-like (Star-like white patch)

Homogeneous areas Brown Periferal Central Total Irreguler Yellowish Periferal Central Total Irreguler Multiple colours 1 Vascular structures Erythema Peripheral Central Total Dotted vessels Hairpin vessels Glomerular vessels Comma vessels Other dermoscopic structures Ring-like structures Ulceration Scales Fissures Exophytic papillary structures Milia-like c

\begin{tabular}{ll}
\hline Pattern & Cases $(\mathrm{n} / \%)$ \\
\hline $1 \mathrm{a}$ & $19(13.4)$ \\
$1 \mathrm{~b}$ & $12(8.5)$ \\
$2 \mathrm{a}$ & $13(9.2)$ \\
$2 \mathrm{~b}$ & $6(4.2)$ \\
$3 \mathrm{a}$ & $8(5.6)$ \\
$3 \mathrm{~b}$ & $2(1.4)$ \\
4 & $8(5.6)$ \\
5 & $2(1.4)$ \\
6 & $15(10.6)$ \\
$7 \mathrm{a}$ & $0(0)$ \\
$7 \mathrm{~b}$ & $7(4.9)$ \\
$8 \mathrm{a}$ & $15(10.6)$ \\
$8 \mathrm{~b}$ & $6(4.2)$ \\
$9 \mathrm{a}$ & $6(4.2)$ \\
$9 \mathrm{~b}$ & $7(4.9)$ \\
10 & $16(11.3)$ \\
\hline
\end{tabular}

Table 2. 
$1 a$

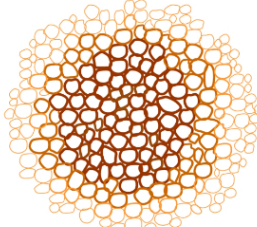

$2 a$

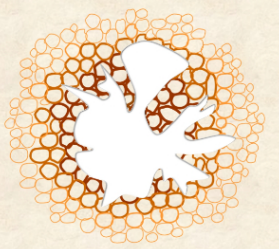

$3 a$

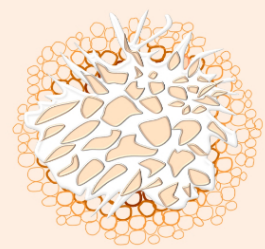

4

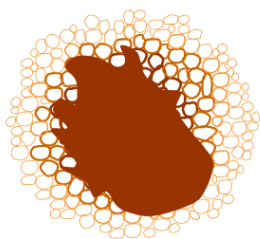

$1 b$

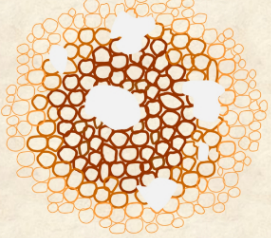

$2 b$

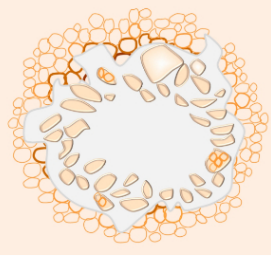

3b

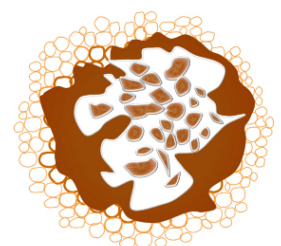

5

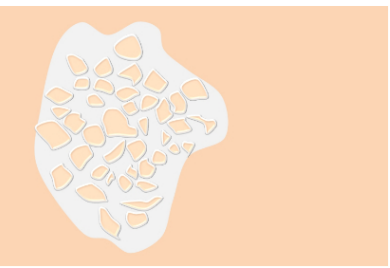




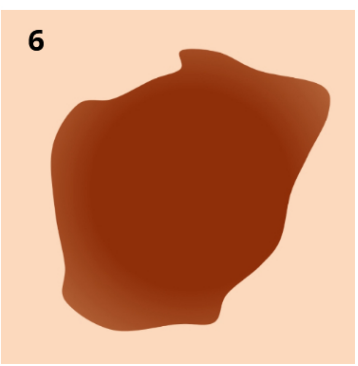

$8 a$

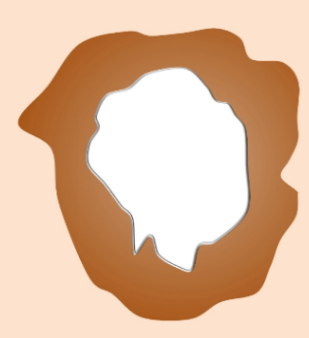

$7 a$

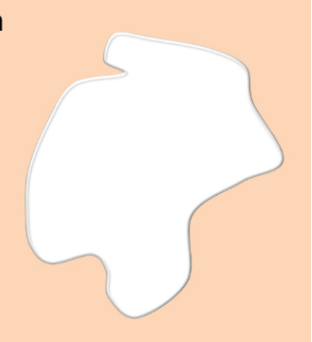

$8 b$

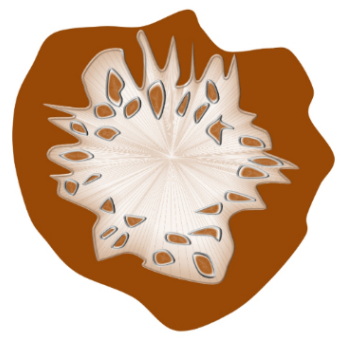

$9 a$

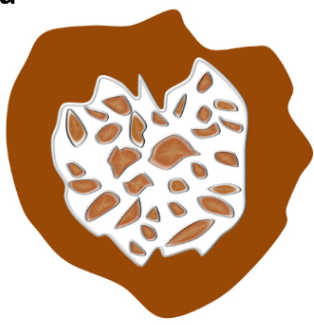

9b

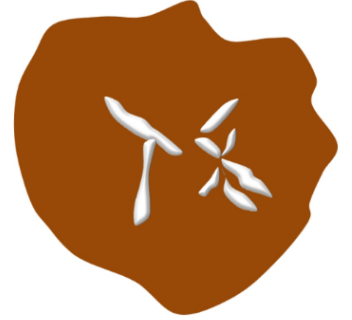

$7 b$

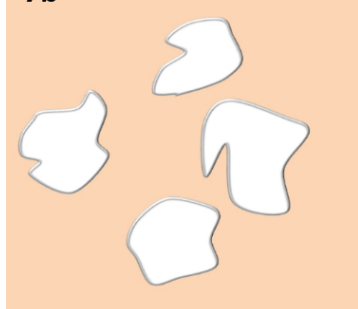

10

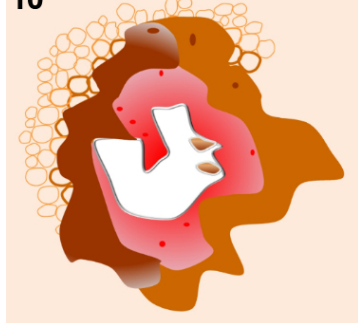



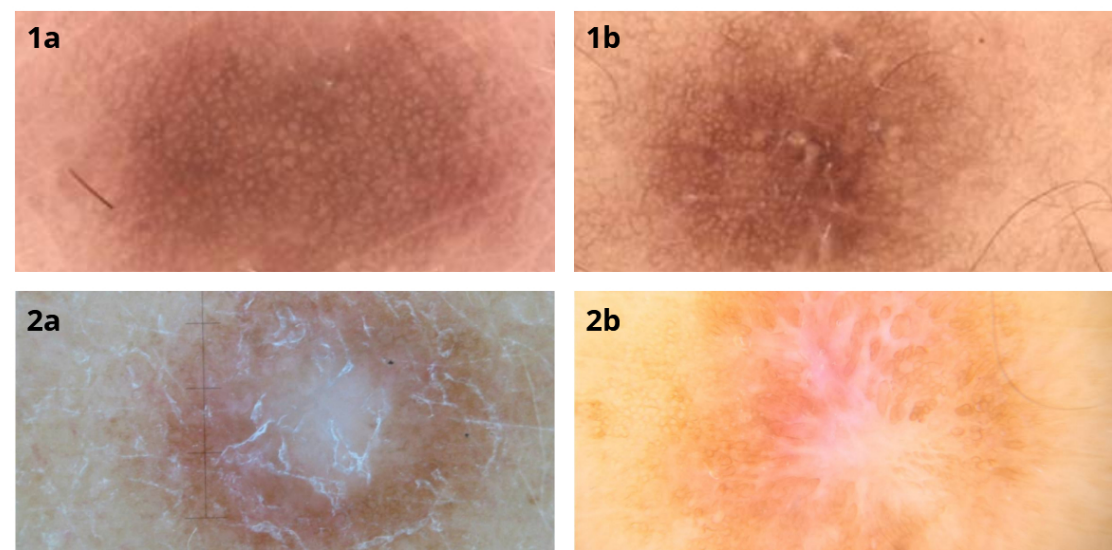

2b
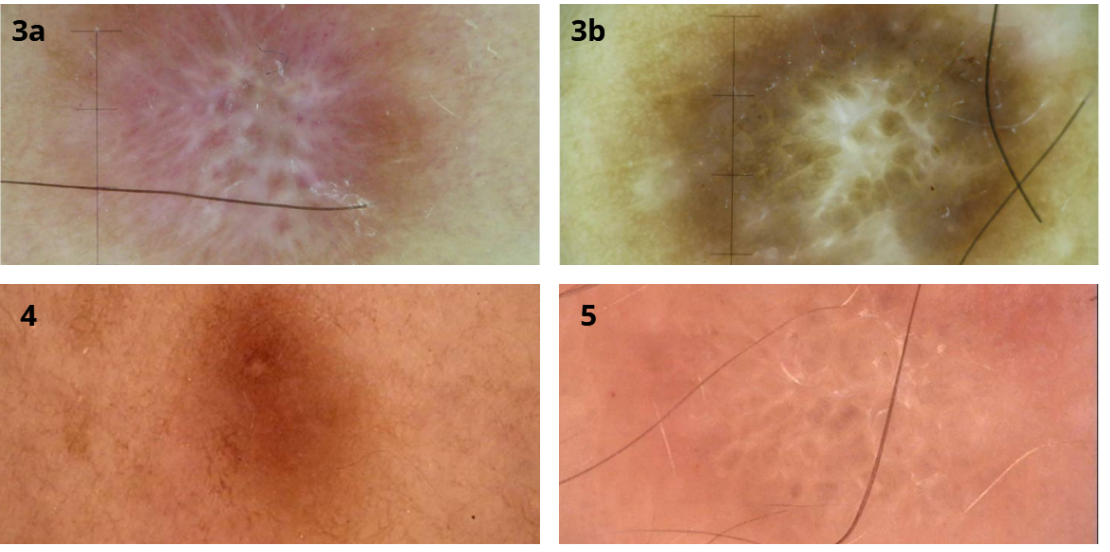


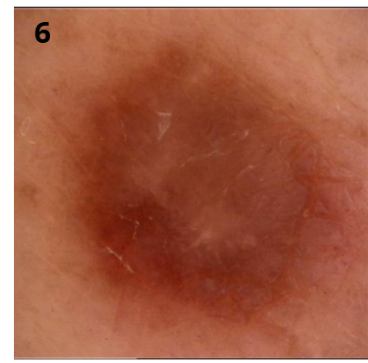

$7 a$

$8 a$

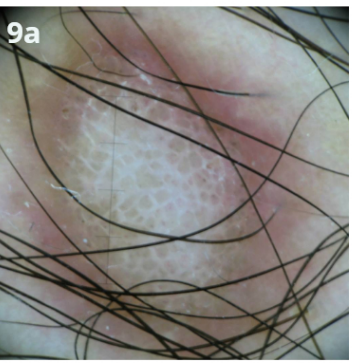

7b

No dermoscopic

image
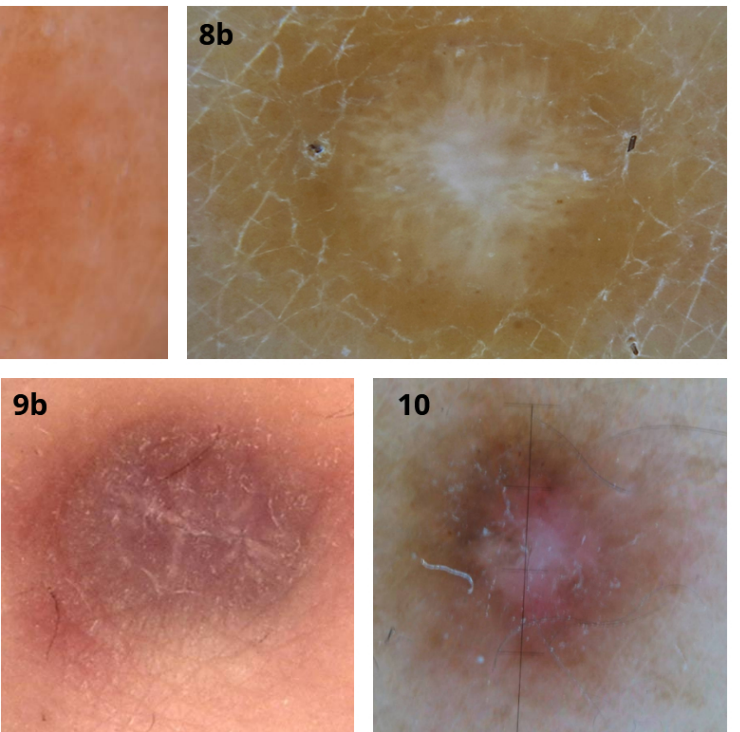

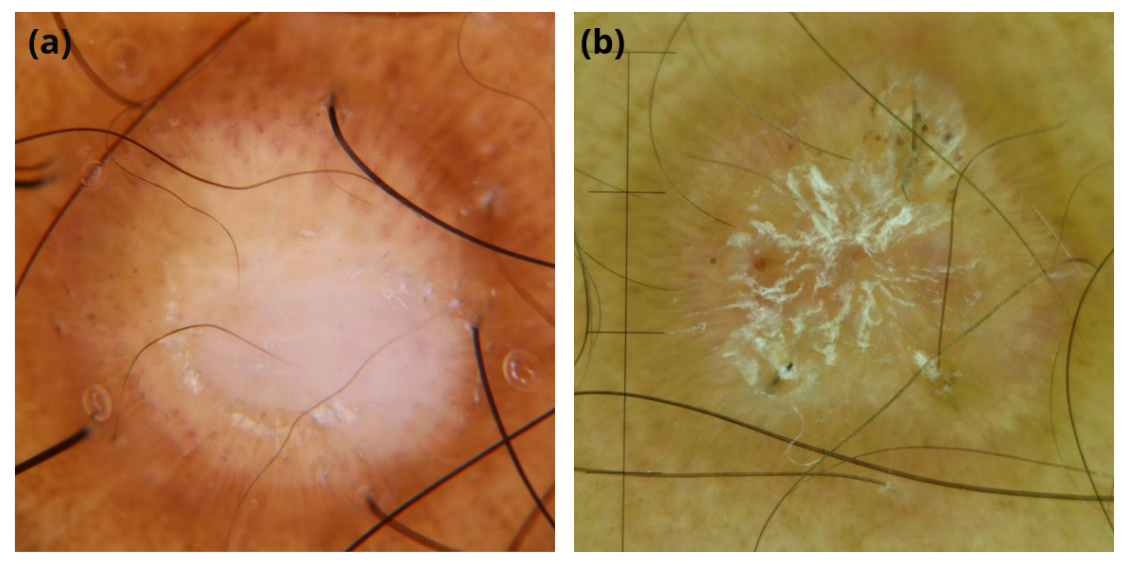

(c) 